\title{
(6) OPEN ACCESS \\ Association between diastolic dysfunction and future atrial fibrillation in the Tromsø Study from 1994 to 2010
}

\author{
Sweta Tiwari, ${ }^{1}$ Henrik Schirmer, ${ }^{2,3}$ Bjarne K Jacobsen, ${ }^{1}$ Laila A Hopstock, ${ }^{1}$ \\ Audhild Nyrnes, ${ }^{1}$ Geir Heggelund ${ }^{2}$ Inger Njølstad, ${ }^{1}$ Ellisiv B Mathiesen, ${ }^{2,4}$ \\ Maja-Lisa Løchen ${ }^{1}$
}

${ }^{1}$ Department of Community Medicine, UiT The Arctic University of Norway, Tromsø, Norway

${ }^{2}$ Department of Clinical Medicine, UiT The Arctic University of Norway, Tromsø, Norway

${ }^{3}$ Department of Cardiology, The University Hospital of North Norway, Tromsø, Norway ${ }^{4}$ Department of Neurology and Neurophysiology, The University Hospital of North Norway, Tromsø, Norway

\section{Correspondence to} Sweta Tiwari, Department of Community Medicine, UiT The Arctic University of Norway, N-9037 Tromsø, Norway; sweta.tiwari@uit.no

Received 2 January 2015 Revised 8 April 2015 Accepted 13 April 2015 Published Online First 13 May 2015

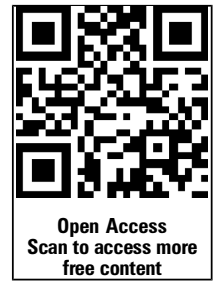

\section{SLinked}

- http://dx.doi.org/10.1136/ heartjnl-2015-307885

\section{CrossMark}

\section{To cite: Tiwari $\mathrm{S}$,}

Schirmer $\mathrm{H}$, Jacobsen BK et al. Heart

2015;101:1302-1308.

\section{ABSTRACT}

Objective To investigate the association between echocardiographic measurements with emphasis on diastolic dysfunction and risk of atrial fibrillation (AF) in a population-based cohort study.

Methods We followed 2406 participants from the Tromsø Study from 1994 to 2010. Left atrial (LA) size and mitral Doppler indices as measured by echocardiography were used for evaluating diastolic dysfunction. Information concerning age, systolic blood pressure, height, heart rate, body mass index, total and high-density lipoprotein cholesterol, self-reported use of alcohol, smoking, coffee, physical activity, antihypertensive treatment, prevalent coronary heart disease, valvular heart disease, heart failure, hypertrophy, diabetes and palpitations were obtained at baseline. The outcome measure was clinical AF, documented by an ECG.

Results AF was detected in 462 subjects (193 women). Mean age at baseline was 62.6 years. Incidence rate of clinical AF was 12.6 per 1000 personyears. In multivariable Cox proportional hazards regression analysis, moderately enlarged LA was associated with $60 \%(95 \% \mathrm{Cl} 1.2$ to 2.0$)$ increased risk of AF. Severely enlarged LA had HR for AF of 4.2 (95\% $\mathrm{Cl} 2.7$ to 6.5$)$ with $\mathrm{p}$ value for linear trend $<0.001$, and the association was similar in both sexes. Abnormal mitral Doppler flow adjusted for predictor variables did not show a statistically significant association with AF risk. However, when LA size was also adjusted for, the risk of $A F$ increased by $30 \%(95 \% \mathrm{Cl} 1.0$ to 1.6).

Conclusions Our findings suggest that enlarged LA as a measure for diastolic dysfunction is a significant risk factor for AF in both sexes, and adding measures of abnormal diastolic flow increased the predictive ability significantly.

\section{INTRODUCTION}

Atrial fibrillation (AF) is a common arrhythmia and is associated with increased mortality and morbidity. $^{1}{ }^{2}$ It increases the risk of stroke and heart failure, affecting quality of life and cognition. ${ }^{23}$

Established risk factors for AF include advancing age, male sex, hypertension, diabetes mellitus, coronary heart disease (CHD), hyperthyroidism, inflammation, obesity, diastolic dysfunction and stroke. $^{4} 5$ The Framingham study found that enlarged left atrial (LA) size is associated with increased risk of AF in both sexes aged 5990 years. ${ }^{6}$ The ARIC study found a higher prevalence of AF in people with dilated LA. ${ }^{7}$ Another study on subjects aged 65 years and above found that LV diastolic dysfunction predicted $\mathrm{AF}^{8}$ The Framingham study confirmed this finding in people with mean age of 75 years. ${ }^{9}$ Among patients with acute myocardial infarction (MI) and reduced LV systolic function, diastolic dysfunction was associated with increased risk of AF. ${ }^{10}$ However, these risk factors need to be validated in large cohorts with longer follow-ups.

Our aim was to investigate the association between echocardiographic measurements with emphasis on diastolic dysfunction and risk of incident clinical AF in the population-based Tromsø Study with 16 years of follow-up.

\section{METHODS}

Study population

The Tromsø Study is a Norwegian prospective cohort study. ${ }^{11}$ It was initiated in 1974 with an emphasis on the epidemiology of cardiovascular diseases. In the fourth survey (Tromsø 4) in 19941995 , all inhabitants 25 years or older were invited and 27158 people (77\% of the eligible population) participated. Among them, all individuals aged 5574 years and 5\%-10\% samples of the other age groups (aged 25-54 and 75-84 years) were invited for a second visit, which included extensive examinations. The 6902 individuals who attended were randomly allocated to one of two lines of examination. One of the lines included echocardiography. ${ }^{12}$ The attendance rate was $88 \% .{ }^{13}$ Figure 1 shows that after exclusion, 2406 participants were included. The survey complies with the Declaration of Helsinki, and was approved by the Regional Committee for Medical and Health Research Ethics, the Data Inspectorate and the Norwegian Directorate of Health.

\section{Baseline characteristics}

Questionnaire data were used for predictor variables on alcohol consumption (no alcohol/low alcohol intake (0-4 times/month)/high alcohol intake $(\geq 5$ times/month)), coffee consumption (cups/day), smoking (current/previous/never), diabetes (yes/no), antihypertensive treatment (current/ previous/never), palpitations (yes/no), prevalent 
Figure 1 Flow chart, the Tromsø Study, 1994-2010. AF, atrial fibrillation.

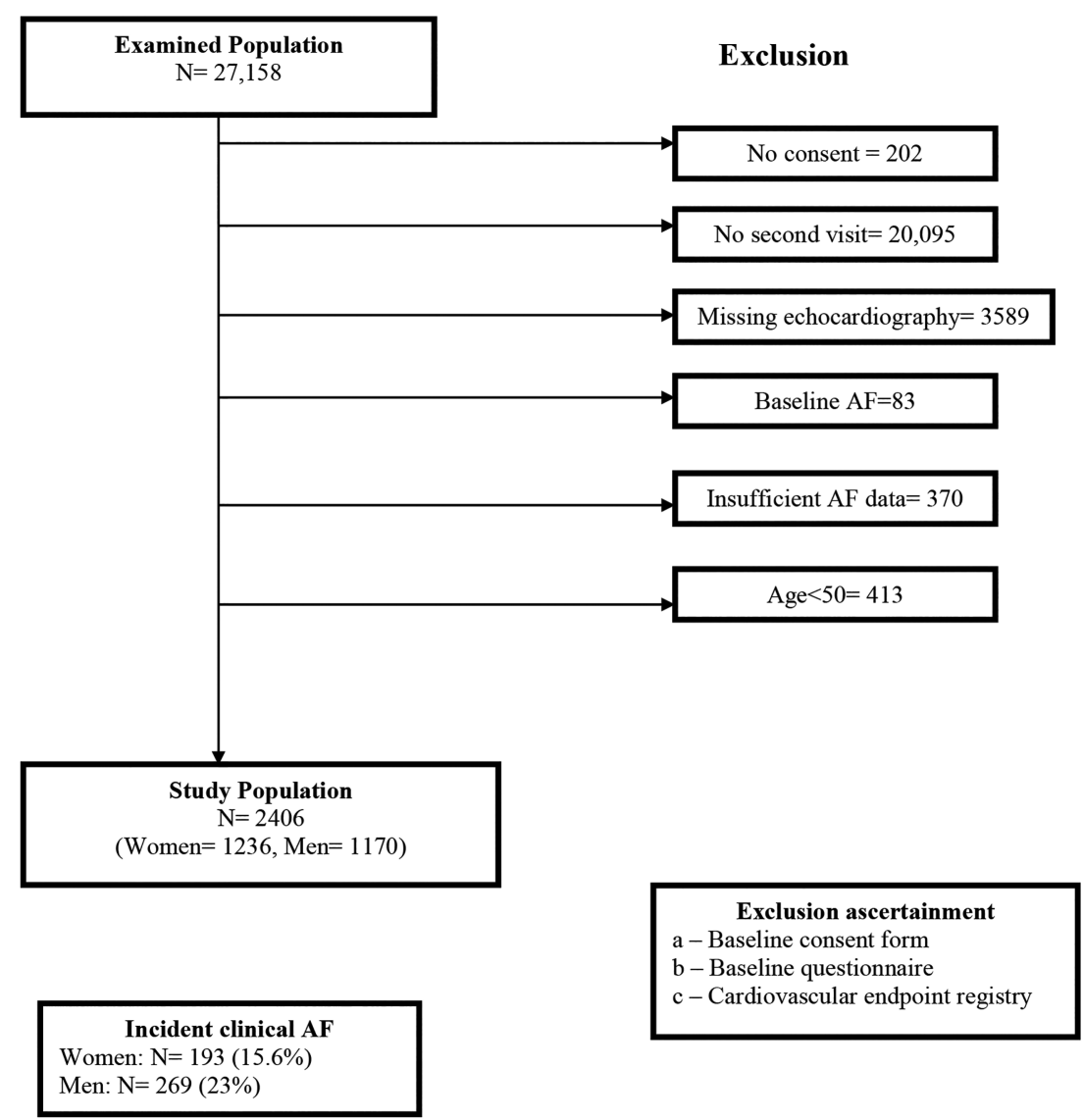

CHD (yes/no), thyroid disease (yes/no) and physical activity (active (weekly exercise with sweating or being out of breath or $\geq 3 \mathrm{~h}$ per week of light exercise without sweating or being out of breath) and inactive ( $<3 \mathrm{~h}$ per week of activity without sweating or being out of breath)). ${ }^{14}$ Prevalent CHD was defined as self-reported previous MI. Body mass index (BMI) was calculated as weight $/$ height ${ }^{2}\left(\mathrm{~kg} / \mathrm{m}^{2}\right)$ and body surface area (BSA) was calculated by $\mathrm{Du}$ Bois formula $\left(\mathrm{BSA}=\left(\right.\right.$ weight $(\mathrm{kg})^{0.425} \times$ height $\left.\left.(\mathrm{cm})^{0.725}\right) \times 0.007184\right)$. Blood pressure and heart rate were recorded three times with $1 \mathrm{~min}$ intervals after $2 \mathrm{~min}$ resting, using an automatic device (Dinamap Vital Signs Monitor 1846, Critikon), and the mean from the second and third reading was used. Hypertension was defined as systolic blood pressure (SBP) $\geq 140 \mathrm{~mm} \mathrm{Hg}$ or diastolic blood pressure $\geq 90 \mathrm{~mm} \mathrm{Hg}$ or use of antihypertensive medication. Non-fasting total cholesterol was analysed by enzymatic methods with commercial kits, and serum high-density lipoprotein cholesterol was measured after the precipitation of low-density lipoprotein with heparin and manganese chloride. All blood sample analyses were performed at the Department of Clinical Chemistry, University Hospital of North Norway.

\section{Echocardiography}

Echocardiography was performed by two expert cardiologists using a VingMed CFM 750 (VingMed Sound A/S, Horten, Norway) with a combined $3.25 \mathrm{MHz}$ mechanical and $2.5 \mathrm{MHz}$ Doppler probe. The examinations were performed using the standard apical and parasternal long and short axis views. Standard 2D-guided M-mode registrations of anteroposterior LA size, internal dimensions of the LV and wall-thickness of the septum and posterior wall were made from leading-edge-toleading-edge convention. ${ }^{15}$
The measurements of peak flow velocity in early diastole (E-wave) and during atrial contraction (A-wave), peak E/A ratio and the E-wave deceleration time (EDT) were done online in one heart cycle. The influence of heart rate was minimised by measuring EDT as the time between the peak E-wave and the upper deceleration slope extrapolated to the zero baselines. ${ }^{13}$ LA size was indexed by BSA. Valvular heart disease was defined as mitral insufficiency grade $3\left(>7 \mathrm{~cm}^{2}\right)$, heart failure as LVEF $<0.5$ and hypertrophy as LV posterior wall end diastole M-mode $>1.4 \mathrm{~cm}$ and/or interventricular septum end diastole $\mathrm{M}$-mode $>1.4 \mathrm{~cm}$.

\section{Reproducibility}

A reproducibility study of the echocardiographic data was performed in a subsample of 58 subjects who were examined twice with 1 -week interval by both cardiologists. For the E-wave, the mean differences \pm SD were $-0.001 \pm 0.117,0.025 \pm 0.096$ and $0.034 \pm 0.078 \mathrm{~m} / \mathrm{s}$ for intraobserver cardiologist 1 , intraobserver cardiologist 2 and interobserver pairs, respectively. For the A-wave, the corresponding values were $-0.008 \pm 0.091,0.031$ \pm 0.156 and $-0.003 \pm 0.084 \mathrm{~m} / \mathrm{s}$. For the E/A ratio, the values were $0.015 \pm 0.29,0.007 \pm 0.28$ and $0.056 \pm 0.19$, and for the deceleration time, $-0.001 \pm 0.034,-0.001 \pm 0.030$ and -0.009 \pm 0.036 s. $^{13}$

\section{Classification of diastolic dysfunction}

We used LA size and mitral Doppler indices for evaluating diastolic dysfunction. Diastolic dysfunction was classified according to current guidelines, ${ }^{16}$ and the cut-off values for E/A ratios and EDT were set with slight adjustments, consistent with the values in a previous community-based study. ${ }^{17}$ 


\section{Mitral Doppler indices}

Mitral Doppler indices are classified according to increasing degree of diastolic dysfunction as follows:

Group I: E/A ratio $0.75-1.5$ and EDT $\geq 140 \mathrm{~ms}$

Group II: E/A ratio $>1.5$ and EDT $>140 \mathrm{~ms}$

Group III : E/A ratio $<0.75$ and any EDT

Group IV: E/A ratio $>0.75$ and EDT $<140 \mathrm{~ms}$

Also, mitral Doppler indices are classified as:

Normal: E/A ratio $0.75-1.5$ and EDT $\geq 140 \mathrm{~ms}$

Abnormal: E/A ratio $<0.75$ or $>1.5$ or EDT $<140 \mathrm{~ms}$

\section{LA size}

LA size was indexed by BSA and categorised into three groups as normal $\left(<2.2 \mathrm{~cm} / \mathrm{m}^{2}\right)$, moderately enlarged $\left(2.2-2.79 \mathrm{~cm} / \mathrm{m}^{2}\right)$ and severely enlarged $\left(\geq 2.8 \mathrm{~cm} / \mathrm{m}^{2}\right){ }^{18}$

\section{Mitral Doppler indices and LA size combined}

Mitral Doppler indices and LA size combined into six groups as follows:

Group 1=LA size $<2.2$ and normal mitral Doppler indices Group 2=LA size $<2.2$ and abnormal mitral Doppler indices Group 3=LA size 2.2-2.79 and normal mitral Doppler indices Group 4=LA size 2.2-2.79 and abnormal mitral Doppler indices

Group 5=LA size $\geq 2.8$ and normal mitral Doppler indices Group $6=$ LA size $\geq 2.8$ and abnormal mitral Doppler indices

\section{Endpoints}

All participants were followed with respect to incident clinical AF documented by an ECG. Norway has a personal identification number that allows exact matching of population register data. The identification number of participants of the 19941995 study was linked to the diagnosis registry at the University Hospital of North Norway (outpatient clinic included) and to the National Causes of Death Registry at Statistics Norway, using the following diagnostic codes: ICD-9 codes 427.0427.99 and ICD-10 codes I47 and I48. For participants with diagnosis of cerebrovascular or cardiovascular events but without a diagnosis of arrhythmia, paper versions of hospital records (used until 2001) for notes on AF were manually searched, and text searches with the term 'atrial fibrillation' were performed in the electronic records. An independent endpoint committee adjudicated the events. All AF types were merged in the analyses. Subjects with transient AF only during acute MI or cardiac surgery and people with AF only in the last week of life were not classified with AF. We followed 2406 participants (figure 1) until the date of first documented AF or date of censoring due to death, migration or end of follow-up on 31 December 2010, whichever came first. Those who had died or emigrated during follow-up were identified through the Population Registry of Norway.

\section{Statistical analysis}

We present the characteristics of the study population with and without AF as means and SDs for continuous variables and proportions of group total for categorical variables. Sex-specific HRs for AF were estimated by multivariable Cox proportional hazards regression models adjusted for age, height, BMI, hypertension, palpitations and diabetes. The variables that were included in the final multivariable model were chosen based on prior scientific knowledge ${ }^{19}$ or because they were significant for either sex in current univariable analyses for atrial size. We tested for interactions between main independent variables (atrial size, mitral Doppler indices group) and sex. We excluded those with colinearity (tolerance $<0.10$ ) from the final model. The variable hypertension (yes/no) was used, and the variable antihypertensive treatment and SBP were not included in the multivariable models as it rendered similar information. Similarly, when colinearity was tested with all the variables, the tolerance was very low for BSA (tolerance $=0.004$; variance inflation factor (VIF) $=234.03$ ); thus, only height was included. Due to few cases in each category, prevalent cardiovascular diseases (CHD, valvular heart disease, hypertrophy and heart failure) were not adjusted for. The proportional hazard assumption was validated with visual inspection of log-minus-log plots. We calculated the c-statistic of the model to predict its clinical usefulness for distinguishing high-risk from low-risk subjects and log-likelihood ratio test to evaluate whether addition of another variable improved the predictive ability significantly. A two-sided $\mathrm{p}$ value $<0.05$ was considered statistically significant. Statistical analysis was performed using STATA V.12 (Stata, College Station, Texas, USA).

\section{RESULTS}

Incident clinical AF was identified in 193 women (15.6\%) and 269 men (23\%) (figure 1). The incidence rate of clinical AF per 1000 person-years was 11.2 in women and 17.5 in men. Table 1 shows the baseline characteristics for subjects with and without AF. Participants developing AF were significantly older, had higher blood pressure, total cholesterol, BMI and BSA, higher prevalence of $\mathrm{CHD}$, diabetes, palpitations, valvular heart disease, heart failure, hypertrophy and taking antihypertensive treatment. They also had higher LV mass, LA size, E/A ratio and deceleration time. Women with subsequent AF consumed little alcohol and were less physically active.

Table 2 gives the HRs for AF according to indexed LA size categories, mitral Doppler groups and for abnormal mitral Doppler flow. The risk of AF increased with increasing atrial size. In multivariable Cox proportional hazards regression analysis (model 2), a moderately enlarged LA was associated with $60 \%$ (95\% CI 1.2 to 2.0) increased risk of AF compared with subjects with normal LA size. In subjects with severely enlarged LA, HR for AF was 4.2 (95\% CI 2.7 to 6.5) compared with subjects with normal LA size ( $\mathrm{p}$ value for linear trend $<0.0001)$. The result was unchanged after adjustment for abnormal mitral Doppler flow (model 3) or mitral Doppler groups (model 4) or when excluding those with baseline CHD, valvular heart disease, heart failure or hypertrophy (model 5). There were no associations between AF and abnormal mitral Doppler flow or AF and mitral Doppler groups (model 2) when LA size was not adjusted for. When LA size was adjusted for (model 4), abnormal mitral Doppler flow was associated with $30 \%$ (95\% CI 1.0 to 1.6) increased risk of AF compared with subjects with normal mitral Doppler flow.

Analysis of c-statistics revealed that the model with LA size and all predictor variables together gave better prediction than a model adjusted for only age and sex (Harrell's c $=0.71$ vs 0.67 , $\mathrm{p}<0.0001)$ or a model with all predictors and mitral Doppler groups/flow (Harrell's $c=0.71$ vs $0.69, \quad p=0.001$ ). The c-statistics comparing the model with only age and sex to a model with all predictors and mitral Doppler groups/flow were Harrell's $c=0.69$ vs $0.67, p=0.001$.

We also calculated the log-likelihood ratio $\chi^{2}$ statistics to compare the predictive ability of the models. When LA size was added to a model with only baseline characteristics, the model improved significantly $(\mathrm{p}<0.0001)$. Comparing baseline characteristics with mitral Doppler flow or mitral Doppler 
Table 1 Unadjusted baseline characteristics of women and men by incident clinical AF during follow-up

\begin{tabular}{|c|c|c|c|c|}
\hline \multirow[b]{2}{*}{ Baseline characteristics } & \multicolumn{2}{|l|}{ Women with } & \multicolumn{2}{|l|}{ Men with } \\
\hline & AF $(n=193)$ & No AF $(n=1043)$ & AF $(n=269)$ & No $A F(n=901)$ \\
\hline Age (years) & $66.4(5.7)$ & $62.5(6.0)$ & $64.2(6.3)$ & $61.5(6.4)$ \\
\hline Diastolic blood pressure $(\mathrm{mm} \mathrm{Hg})$ & $86.2(14.4)$ & $82.2(12.3)$ & $86.2(12.5)$ & $85.4(11.4)$ \\
\hline Heart rate (beats/min) & $73.9(13.7)$ & $75.2(11.6)$ & $69.9(13.2)$ & $71.4(12.5)$ \\
\hline Height $(\mathrm{cm})$ & $161.6(6.7)$ & $161.1(6.1)$ & $175.3(7.1)$ & $174.7(6.6)$ \\
\hline $\operatorname{BSA}\left(m^{2}\right)$ & $1.8(0.2)$ & $1.7(0.1)$ & $2.0(0.2)$ & $1.9(0.2)$ \\
\hline Total cholesterol (mmol/L) & $7.13(1.13)$ & $7.08(1.24)$ & $6.58(1.14)$ & $6.57(1.19)$ \\
\hline HDL cholesterol (mmol/L) & $1.67(0.45)$ & $1.68(0.42)$ & $1.40(0.39)$ & $1.44(0.40)$ \\
\hline \multicolumn{5}{|l|}{ Alcohol, \% (n) } \\
\hline No alcohol & $37.2(71)$ & $30.4(315)$ & $13.8(37)$ & $13.6(122)$ \\
\hline Low alcohol intake (0-4 times/month) & $61.3(117)$ & $61.8(641)$ & $65.7(176)$ & $65.4(587)$ \\
\hline Current smoking & $25.9(50)$ & $29.4(307)$ & $28.6(77)$ & $35.4(319)$ \\
\hline Coffee (cups/day) & $4.9(2.4)$ & $4.8(2.7)$ & $5.7(3.6)$ & $6.1(3.7)$ \\
\hline Physical activity, \% (n) & $43.5(84)$ & $53.4(557)$ & $66.5(179)$ & $67.4(607)$ \\
\hline Hypertension, \% (n) & $80.8(156)$ & $58.5(610)$ & $70.6(190)$ & $60.8(548)$ \\
\hline Antihypertensive treatment, \% (n) & $25.9(50)$ & $12.2(127)$ & $22.4(60)$ & $10.3(92)$ \\
\hline Coronary heart disease, \% (n) & $6.3(12)$ & $2.6(27)$ & $15.3(41)$ & $6.6(59)$ \\
\hline Valvular heart disease, \% (n) & $1.0(2)$ & $0.3(3)$ & $1.1(3)$ & $0.0(0)$ \\
\hline Heart failure, \% (n) & $0.7(1)$ & $0.3(3)$ & $4.3(9)$ & $1.5(11)$ \\
\hline Hypertrophy, \% (n) & $2.0(3)$ & $0.7(6)$ & $3.8(8)$ & $2.4(18)$ \\
\hline Diabetes, \% (n) & $5.2(10)$ & $2.4(25)$ & $5.2(14)$ & $2.6(23)$ \\
\hline Palpitations, \% (n) & $38.1(56)$ & $29.7(260)$ & $24.4(59)$ & $15.0(124)$ \\
\hline Group I & 71.7 (137) & $75.5(773)$ & $69.2(182)$ & 78.5 (695) \\
\hline Group II & $2.6(5)$ & $2.7(28)$ & $4.9(13)$ & $3.8(34)$ \\
\hline Group III & $22.0(42)$ & 19.4 (199) & $24.0(63)$ & $15.9(141)$ \\
\hline Group IV & $3.7(7)$ & $2.3(24)$ & $1.9(5)$ & $1.7(15)$ \\
\hline $\begin{array}{l}\text { Abnormal mitral Doppler flow } \\
\text { Group II-IV, \% (n) }\end{array}$ & $28.3(54)$ & $24.5(251)$ & $30.8(81)$ & $21.5(190)$ \\
\hline
\end{tabular}

groups added to it, the $\mathrm{p}$ values were 0.1 in both situations. Thus, the model with LA size predicted AF better than the other models.

Figure 2 shows the Kaplan-Meier curves for AF among different categories of LA size and mitral Doppler indices. Those with larger LA size and abnormal mitral Doppler indices have increased risk of AF. When these two risk factors were combined, the risk of AF increased with increasing LA size and abnormal mitral Doppler indices (figure 3). We combined group 5 and 6 due to few cases of AF in each category.

Table 3 shows the sex-specific HRs for AF according to LA size, and mitral Doppler flow and LA size combined. In subjects with severely enlarged LA, HRs for AF were 4.5 (95\% CI 2.6 to $8.0)$ in women and $3.6(95 \%$ CI 1.7 to 7.5$)$ in men compared with subjects with normal LA size ( $\mathrm{p}$ value for linear trend $<0.0001$ ). The associations were similar in both sexes ( $\mathrm{p}$ value for interaction $=0.28$ ). Adjustment for covariates in a multivariable model (model 2) increased HRs, whereas in model 3, excluding participants with baseline CHD, valvular heart disease, heart failure or hypertrophy, attenuated the strength of the relationship slightly in both women and men. Model 4 shows the HRs for AF according to mitral Doppler flow and LA size combined. In this model, the risk of AF increased with increasing atrial size and abnormal mitral Doppler flow compared with normal mitral Doppler flow. In multivariable Cox proportional hazards regression analysis (model 5), in subjects with severely enlarged LA and abnormal mitral Doppler flow, HR for AF was 3.7 (95\% CI 1.6 to 8.7) compared with those 
Table 2 HR $(95 \% \mathrm{Cl})$ for atrial fibrillation according to indexed left atrial (LA) size, mitral Doppler groups and mitral Doppler flow

\begin{tabular}{|c|c|c|c|c|c|}
\hline All & Model 1 & Model 2 & Model 3 & Model 4 & Model 5 \\
\hline \multicolumn{6}{|l|}{ Indexed LA size } \\
\hline $1(<2.2)$ & 1 (Ref.) & 1 (Ref.) & 1 (Ref.) & 1 (Ref.) & 1 (Ref.) \\
\hline $2(2.2-2.79)$ & 1.4 (1.1 to 1.7$)$ & 1.6 (1.2 to 2.0$)$ & 1.6 (1.3 to 2.0$)$ & 1.6 (1.2 to 2.0$)$ & 1.4 (1.1 to 1.8$)$ \\
\hline $3(\geq 2.8)$ & $\begin{array}{l}3.1(2.1 \text { to } 4.4 \text { ) } \\
\text { ( } p \text { for trend }<0.0001 \text { ) }\end{array}$ & $\begin{array}{l}4.2(2.7 \text { to } 6.5) \\
\text { ( } p \text { for trend }<0.0001)\end{array}$ & $\begin{array}{l}4.2(2.7 \text { to } 6.5) \\
\text { ( } p \text { for trend }<0.0001)\end{array}$ & $\begin{array}{l}4.1(2.6 \text { to } 6.4) \\
\text { ( } p \text { for trend }<0.0001 \text { ) }\end{array}$ & $\begin{array}{l}4.0(2.5 \text { to } 6.4) \\
\text { ( } p \text { for trend }<0.0001 \text { ) }\end{array}$ \\
\hline \multicolumn{6}{|l|}{ Mitral Doppler groups } \\
\hline Group I & 1 (Ref.) & 1 (Ref.) & 1 (Ref.) & - & 1 (Ref.) \\
\hline Group II & 1.6 (1.0 to 2.7$)$ & 1.8 (1.1 to 3.0$)$ & 1.5 (0.9 to 2.6$)$ & & $1.6(0.9$ to 2.9$)$ \\
\hline Group III & 1.1 (0.9 to 1.4$)$ & 1.1 (0.9 to 1.4$)$ & $1.2(0.9$ to 1.6$)$ & & 1.1 (0.9 to 1.5$)$ \\
\hline Group IV & $\begin{array}{l}1.7(1.0 \text { to } 3.0) \\
\text { ( } p \text { Value for trend } 0.103 \text { ) }\end{array}$ & $\begin{array}{l}1.5(0.8 \text { to } 3.1) \\
\text { ( } p \text { Value for trend } 0.141 \text { ) }\end{array}$ & $\begin{array}{l}1.5(0.7 \text { to } 2.9) \\
\text { ( } p \text { Value for trend } 0.051 \text { ) }\end{array}$ & & $\begin{array}{l}1.2(0.5 \text { to } 3.0) \\
\text { ( } p \text { Value for trend } 0.240 \text { ) }\end{array}$ \\
\hline $\begin{array}{l}\text { Abnormal mitral Doppler flow } \\
\text { (Group II-IV) }\end{array}$ & $\begin{array}{l}1.2(1.0 \text { to } 1.5) \\
\text { ( } p \text { Value } 0.081)\end{array}$ & $\begin{array}{l}1.2(1.0 \text { to } 1.5) \\
\text { ( } p \text { Value } 0.086)\end{array}$ & - & $\begin{array}{l}1.3(1.0 \text { to } 1.6) \\
\text { ( } p \text { Value } 0.034)\end{array}$ & $\begin{array}{l}1.2(0.9 \text { to } 1.5) \\
\text { ( } p \text { Value } 0.159)\end{array}$ \\
\hline \multicolumn{6}{|c|}{$\begin{array}{l}\text { The Tromsø Study, 1994-2010. } \\
\text { Model 1: adjusted for age and sex. } \\
\text { Model 2: as Model 1+height, body mass index, hypertension, diabetes, palpitation. } \\
\text { Model 3: as Model 2+abnormal mitral Doppler flow (only in indexed LA size), LA size (only in mitral Doppler groups and flow). } \\
\text { Model 4: as Model 2+mitral Doppler groups (only in indexed LA size) or LA size (only in mitral Doppler groups and flow). } \\
\text { Model 5: as Model 2, participants with coronary heart disease, valvular heart disease, heart failure or hypertrophy at baseline excluded. } \\
\text { Mitral Doppler groups: Group I E/A ratio } 0.75-1.5 \text { and E-wave deceleration time (EDT }>140 \mathrm{~ms} \text {; group II E/A ratio }>1.5 \text { and EDT }>140 \text { ms; group III E/A ratio }<0.75 \text { and any EDT; group } \\
\text { IV E/A ratio }>0.75 \text { and EDT }<140 \mathrm{~ms} \text {. } \\
\text { Ref; reference. }\end{array}$} \\
\hline
\end{tabular}

with normal LA size and mitral Doppler flow. We did not perform sex-specific analyses due to few cases of AF in each category. The predicted c-statistic for model 5 compared with the

A

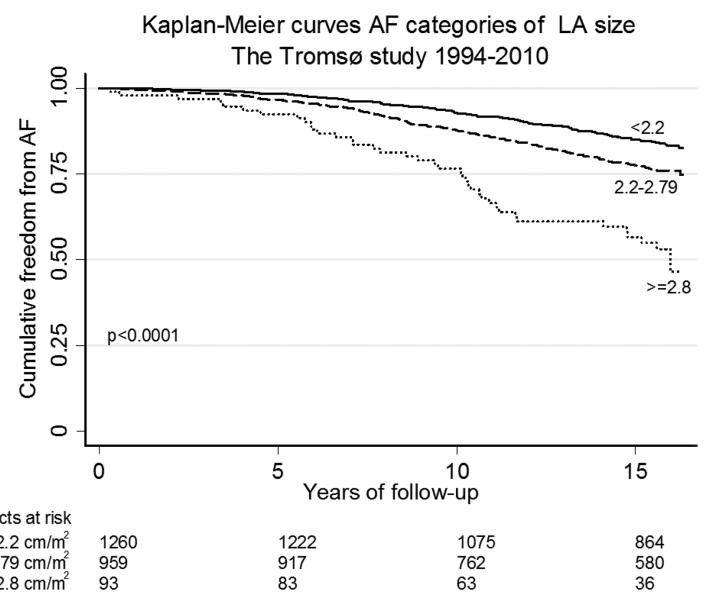

B Kaplan-Meier curves for AF categories of diastolic dysfunction

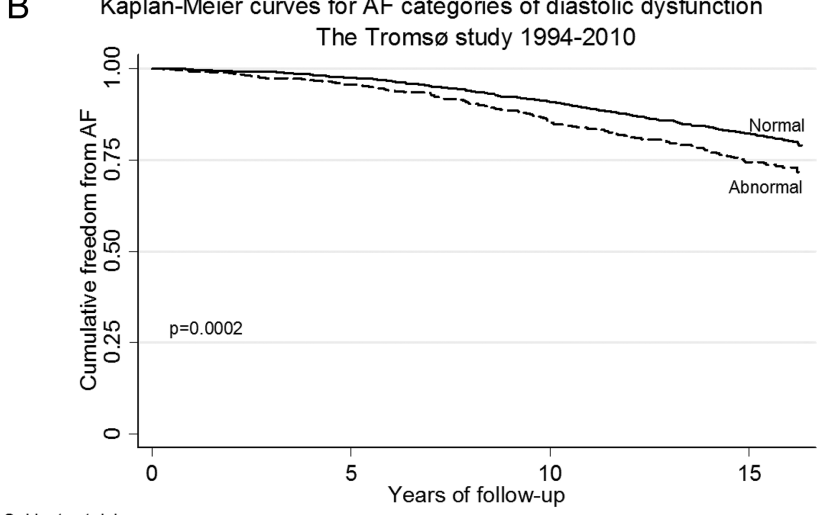

Subjects at risk

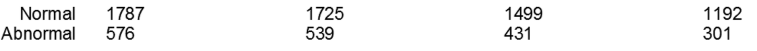

Figure 2 Kaplan-Meier curves for atrial fibrillation (AF): (a) categories of left atrial (LA) size and (b) categories of mitral Doppler indices. The Tromsø Study, 1994-2010. model with only age and sex had better predictive ability (Harrell's c $=0.71$ vs $0.67, p<0.0001$ ). Also, log-likelihood ratio $\chi^{2}$ statistics comparing the model with the combined variable (model 5) with a model comprising only baseline characteristics (age, sex, height, BMI, hypertension, diabetes and palpitation), the model with the combined variable gave a significantly better fit $(\mathrm{p}<0.0001)$.

\section{DISCUSSION}

In this population-based study, we found that the risk of clinical AF increased with increasing LA size in both sexes when adjusted for other known risk factors. There was an increased risk of $\mathrm{AF}$ among subjects with abnormal mitral Doppler indices

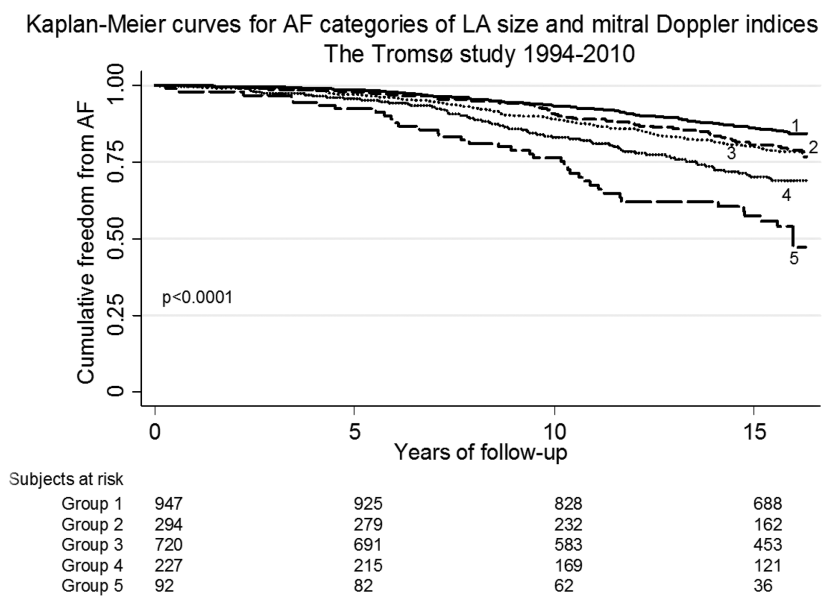

Figure 3 Kaplan-Meier curves for atrial fibrillation (AF) categories of left atrial (LA) size and mitral Doppler indices combined. The Tromsø Study, 1994-2010. Group 1=LA size $<2.2 \mathrm{~cm} / \mathrm{m}^{2}$ and normal mitral Doppler indices ( $\mathrm{n}=947)$. Group 2=LA size $<2.2 \mathrm{~cm} / \mathrm{m}^{2}$ and abnormal mitral Doppler indices $(n=294)$. Group 3=LA size $2.2-2.79 \mathrm{~cm} / \mathrm{m}^{2}$ and normal mitral Doppler indices $(n=720)$. Group 4=LA size 2.2$2.79 \mathrm{~cm} / \mathrm{m}^{2}$ and abnormal mitral Doppler indices ( $\left.n=227\right)$. Group $5=L A$ size $\geq 2.8 \mathrm{~cm} / \mathrm{m}^{2}$ and normal/abnormal mitral Doppler indices ( $\left.n=92\right)$. 
Table 3 Sex-specific HR $(95 \% \mathrm{Cl})$ for atrial fibrillation according to left atrial (LA) size, and mitral Doppler flow and left atrial size combined

\begin{tabular}{|c|c|c|c|c|}
\hline & \multicolumn{3}{|c|}{ Indexed left atrial size } & \multirow[b]{2}{*}{$p$ Value for trend } \\
\hline & $1(<2.2)$ & 2 (2.2 to 2.79$)$ & $3(\geq 2.8)$ & \\
\hline \multicolumn{5}{|l|}{ Women } \\
\hline Events ( $n=177$ ) & $n=61$ & $\mathrm{n}=89$ & $n=27$ & \\
\hline Model 1 & 1 (Ref.) & 1.5 (1.1 to 2.1$)$ & $3.0(1.9$ to 4.9$)$ & $<0.0001$ \\
\hline Model 2 & 1 (Ref.) & 1.6 (1.1 to 2.3$)$ & 4.5 (2.6 to 8.0$)$ & $<0.0001$ \\
\hline Model 3 & 1 (Ref.) & 1.5 (1.0 to 2.3$)$ & $4.4(2.4$ to 8.0$)$ & $<0.0001$ \\
\hline \multicolumn{5}{|l|}{ Men } \\
\hline Events ( $n=259$ ) & $n=128$ & $n=119$ & $n=12$ & \\
\hline Model 1 & 1 (Ref.) & 1.4 (1.1 to 1.8$)$ & 3.0 (1.6 to 5.5$)$ & 0.001 \\
\hline Model 2 & 1 (Ref.) & 1.5 (1.2 to 2.0$)$ & 3.6 (1.7 to 7.5$)$ & $<0.0001$ \\
\hline Model 3 & 1 (Ref.) & $1.3(1.0$ to 1.8$)$ & $3.3(1.3$ to 8.3$)$ & 0.024 \\
\hline \multicolumn{5}{|l|}{ All } \\
\hline Events ( $n=436$ ) & $\mathrm{n}=189$ & $\mathrm{n}=208$ & $n=39$ & \\
\hline \multicolumn{5}{|l|}{ Model 4} \\
\hline \multicolumn{5}{|l|}{ Mitral Doppler flow } \\
\hline Normal & 1 (Ref.) & $1.4(1.1$ to 1.7$)$ & 3.1 (2.1 to 4.8$)$ & $<0.0001^{*}$ \\
\hline Abnormal & $1.2(0.9$ to 1.7$)$ & 1.8 (1.3 to 2.4$)$ & 3.5 (1.8 to 6.7$)$ & \\
\hline \multicolumn{5}{|l|}{ Model 5} \\
\hline \multicolumn{5}{|l|}{ Mitral Doppler flow } \\
\hline Normal & 1 (Ref.) & 1.5 (1.2 to 2.0$)$ & 4.8 (2.9 to 7.8$)$ & $<0.0001^{*}$ \\
\hline Abnormal & 1.3 (0.9 to 1.8$)$ & 2.1 (1.5 to 3.0$)$ & 3.7 (1.6 to 8.7$)$ & \\
\hline \multicolumn{5}{|c|}{$\begin{array}{l}\text { The Tromsø Study, 1994-2010. } \\
\text { * } \mathrm{p} \text { Value for trend is calculated for six groups of LA size and mitral Doppler flow combined. Description can be found in the Methods section. } \\
\text { Model 1: adjusted for age and sex (not in sex-specific models). } \\
\text { Model 2: as Model 1+height, body mass index, hypertension, diabetes, palpitation. } \\
\text { Model 3: as Model 2, participants with coronary heart disease, valvular heart disease, heart failure or hypertrophy at baseline excluded. } \\
\text { Model 4: as Model 1. } \\
\text { Model 5: as Model 2. } \\
\text { Mitral Doppler flow—-normal: E/A ratio } 0.75-1.5 \text { and E-wave deceleration time (EDT }>140 \text { ms; abnormal: E/A ratio }<0.75 \text { or }>1.5 \text { or EDT }<140 \text { ms. } \\
\text { Ref; reference. }\end{array}$} \\
\hline
\end{tabular}

accompanied by enlarged LA. However, there was no association between unadjusted diastolic dysfunction based on mitral Doppler indices and AF risk.

The incidence rate of clinical AF was higher in our study compared with another study done in a similar setting, which could be due to slightly older participants. ${ }^{19}$

\section{Left atrium size}

Some previous studies have found higher risk of AF among those with larger LA. ${ }^{6} \quad 8{ }^{20-24}$ A cross-sectional study showed higher prevalence of AF (15\%) among those with dilated LA compared with those with normal-sized LA $(11 \%) .^{7}$ LA size does not change with ageing, thus enlargement is an expression of pathology. ${ }^{25}$ LA enlargement is due to the change in filling dynamics associated with abnormal $\mathrm{LV}$ relaxation. This results in decreased passive emptying volume from the LA to the LV and decreased direct flow volume from pulmonary veins into the LV in early diastole. ${ }^{21}$ To compensate, active LA contraction is enhanced, increasing the active emptying volume in late diastole. ${ }^{21}$ This preserves LV stroke volume, but it also enlarges the LA predisposing to $\mathrm{AF}^{21}$

A prospective study showed that indexing LA by height was non-significantly better than BSA. We have indexed LA by BSA, as there are no established cut-off values for indexation by height. ${ }^{26}$

\section{Mitral Doppler indices}

We found no independent association between increasing degree of diastolic dysfunction based on mitral Doppler indices and AF. This is in contrast to a study from Minnesota, with participants aged 65 years or older, which found a positive relationship between mitral Doppler indices and AF risk. ${ }^{8}$ Age differences may explain the divergent results, as our study was performed among people 50 years and above. The classification of diastolic dysfunction may be less precise in the middle age groups as the $\mathrm{E} / \mathrm{A}$ ratio is high and DT is low in young and middle-aged adults compared with older people. ${ }^{13} 27$ A study among patients post MI with depressed LV systolic function also found higher risk of AF with diastolic dysfunction. ${ }^{10}$

Our finding of an increased AF risk among subjects with abnormal mitral Doppler flow accompanied by enlarged LA is supported by others. ${ }^{21} 28$ The mitral Doppler indices reflect a snapshot of diastolic function, and the pattern can change if loading condition changes whereas LA size reflects the chronicity of the loading conditions. Thus, LA size provides a longterm view of the condition regardless of loading condition during examination. ${ }^{29}$ LA size increases with worsening diastolic dysfunction, independent of other risk factors. ${ }^{30}$

\section{Strengths}

Our study was performed in a large population-based cohort of both sexes 50 years and older, with a high attendance rate and a long follow-up. Previous studies have been performed in subgroups of elderly subjects with prevalent disease or with shorter follow-up. ${ }^{6-10}$

Another strength was the case validation and search methods. The hospital discharge list may be incomplete. Through systematic hospital record search, we found people who were not registered with AF, but where ECG or medical texts 
documented AF. A reproducibility study was performed to validate echocardiographic data.

\section{Limitations}

Although a detailed search method was used, there may be people with undiagnosed AF. Subjects with asymptomatic or paroxysmal AF often fail to get their arrhythmia documented or are never hospitalised. The Tromsø Study did not acquire data on tissue Doppler or mitral Doppler during Valsalva manoeuvre, and LA size was best evaluated with estimation of volume. ${ }^{28}$ Probably, this attenuated our HR estimates. The external validity refers to Caucasians and may not be generalisable to others.

\section{CONCLUSION}

Enlarged LA as a measure of diastolic dysfunction was independently associated with an increased risk of clinical AF in both sexes, and adding measures of abnormal diastolic flow increased the predictive ability significantly.

\section{Key messages}

\section{What is already known on this subject?}

Left atrial (LA) size and mitral Doppler indices have been associated with atrial fibrillation (AF) in subgroups of elderly and subjects with prevalent disease and in studies with relatively short follow-up.

\section{What might this study add?}

This study provides sex-specific long-term results for the association between LA and AF in a large population-based cohort. Men and women with a severely enlarged LA had a fourfold increased risk of developing AF. Adding measures of abnormal diastolic flow increased the predictive ability significantly, but unadjusted mitral Doppler indices did not show any association with AF risk.

\section{How might this impact on clinical practice?}

Enlarged LA is a sign of chronically increased LA pressure and a risk factor for $\mathrm{AF}$ and thereby for subsequent stroke. A closer clinical monitoring of patients with a $\mathrm{CHA}_{2} \mathrm{DS}_{2}$-VASc score $>1$ together with good patient education of the clinical signs of $A F$ is probably the appropriate measure to improve stroke prophylaxis.

Contributors ST: data analysis and writing of manuscript. HS and M-LL: conception and design of study, data collection and interpretation and revision of manuscript. BKJ, LAH and GH: data interpretation and revision of manuscript. $A N$, IN and EBM: data collection and interpretation and revision of manuscript.

\section{Competing interests None declared.}

Ethics approval Regional Committee for Medical and Health Research Ethics, the Data Inspectorate and the Norwegian Directorate of Health.

Provenance and peer review Not commissioned; externally peer reviewed.

Open Access This is an Open Access article distributed in accordance with the Creative Commons Attribution Non Commercial (CC BY-NC 4.0) license, which permits others to distribute, remix, adapt, build upon this work non-commercially, and license their derivative works on different terms, provided the original work is properly cited and the use is non-commercial. See: http://creativecommons.org/ licenses/by-nc/4.0/

\section{REFERENCES}

1 Benjamin EJ, Wolf PA, D'Agostino RB, et al. Impact of atrial fibrillation on the risk of death: the Framingham Heart Study. Circulation 1998;98:946-52.

2 Magnani JW, Rienstra M, Lin $\mathrm{H}$, et al. Atrial fibrillation: current knowledge and future directions in epidemiology and genomics. Circulation 2011;124:1982-93.
3 Stewart S, Hart CL, Hole DJ, et al. A population-based study of the long-term risks associated with atrial fibrillation: 20-year follow-up of the Renfrew/Paisley study. Am J Med 2002;113:359-64.

4 Gersh BJ, Tsang TSM, Barnes ME, et al. The changing epidemiology of non-valvular atrial fibrillation: the role of novel risk factors. Eur Heart J Supp/ 2005;7(Suppl C): C5-11.

5 Ahmad Y, Lip GY, Lane DA. Recent developments in understanding epidemiology and risk determinants of atrial fibrillation as a cause of stroke. Can J Cardiol 2013;29(7 Suppl):S4-13.

6 Vaziri SM, Larson MG, Benjamin EJ, et al. Echocardiographic predictors of nonrheumatic atrial fibrillation. The Framingham Heart Study. Circulation 1994;89:724-30.

7 Qureshi W, Soliman EZ, Solomon SD, et al. Risk factors for atrial fibrillation in patients with normal versus dilated left atrium (from the Atherosclerosis Risk in Communities Study). Am J Cardiol 2014;114:1368-72.

8 Tsang TS, Gersh BJ, Appleton CP, et al. Left ventricular diastolic dysfunction as a predictor of the first diagnosed nonvalvular atrial fibrillation in 840 elderly men and women. J Am Coll Cardiol 2002;40:1636-44.

9 Vasan RS, Larson MG, Levy D, et al. Doppler transmitral flow indexes and risk of atrial fibrillation (the Framingham Heart Study). Am J Cardiol 2003;91:1079-83.

10 Jons $C$, Joergensen RM, Hassager $C$, et al. Diastolic dysfunction predicts new-onset atrial fibrillation and cardiovascular events in patients with acute myocardial infarction and depressed left ventricular systolic function: a CARISMA substudy. European J Echocardiogr 2010;11:602-7.

11 Jacobsen BK, Eggen AE, Mathiesen EB, et al. Cohort profile: the Tromso Study. Int J Epidemiol 2012:41:961-7.

12 Lindekleiv H, Løchen ML, Mathiesen EB, et al. Echocardiographic screening of the general population and long-term survival: a randomized clinical study. JAMA Intern Med 2013;173:1592-8.

13 Schirmer H, Lunde P, Rasmussen K. Mitral flow derived Doppler indices of left ventricular diastolic function in a general population; the Tromso study. Eur Heart J 2000;21:1376-86.

14 Arntzen KA, Schirmer $\mathrm{H}$, Wilsgaard T, et al. Moderate wine consumption is associated with better cognitive test results: a 7 year follow up of 5033 subjects in the Tromso Study. Acta Neurol Scand Supp/ 2010;122:23-9.

15 O'Rourke RA, Hanrath P, Henry WN, et al. Report of the Joint International Society and Federation of Cardiology/World Health Organization Task Force on Recommendations for Standardization of Measurements from M-mode Echocardiograms. Circulation 1984;69:854A-7A.

16 Nagueh SF, Appleton CP, Gillebert TC, et al. Recommendations for the evaluation of left ventricular diastolic function by echocardiography. Eur J Echocardiogr 2009;10:165-93.

17 Redfield MM, Jacobsen SJ, Burnett JC Jr, et al. Burden of systolic and diastolic ventricular dysfunction in the community: appreciating the scope of the heart failure epidemic. JAMA 2003;289:194-202.

18 Lang RM, Bierig M, Devereux RB, et al. Recommendations for chamber quantification. Eur J Echocardiogr 2006;7:79-108.

19 Nyrnes A, Mathiesen EB, Njølstad I, et al. Palpitations are predictive of future atrial fibrillation. An 11-year follow-up of 22,815 men and women: the Tromso Study. Eur J Prev Cardiol 2013;20:729-36.

20 Bangalore S, Yao SS, Chaudhry FA. Role of left atrial size in risk stratification and prognosis of patients undergoing stress echocardiography. J Am Coll Cardiol 2007:50:1254-62.

21 Tsang TS, Barnes ME, Gersh BJ, et al. Risks for atrial fibrillation and congestive heart failure in patients $>/=65$ years of age with abnormal left ventricular diastolic relaxation. Am J Cardiol 2004;93:54-8.

22 Patton KK, Ellinor PT, Heckbert SR, et al. N-terminal pro-B-type natriuretic peptide is a major predictor of the development of atrial fibrillation: the Cardiovascular Health Study. Circulation 2009;120:1768-74.

23 Rosenberg MA, Gottdiener JS, Heckbert SR, et al. Echocardiographic diastolic parameters and risk of atrial fibrillation: the Cardiovascular Health Study. Eur Heart J 2012;33:904-12.

24 Fatema K, Barnes ME, Bailey KR, et al. Minimum vs. maximum left atrial volume for prediction of first atrial fibrillation or flutter in an elderly cohort: a prospective study. Eur J Echocardiogr 2009;10:282-6.

25 Thomas L, Levett K, Boyd A, et al. Compensatory changes in atrial volumes with normal aging: is atrial enlargement inevitable? J Am Coll Cardiol 2002;40:1630-5.

26 Armstrong AC, Liu K, Lewis CE, et al. Left atrial dimension and traditional cardiovascular risk factors predict 20-year clinical cardiovascular events in young healthy adults: the CARDIA study. Eur Heart J Cardiovasc Imaging 2014;15:893-9.

27 Mottram PM, Marwick TH. Assessment of diastolic function: what the general cardiologist needs to know. Heart 2005;91:681-95.

28 Tsang TS, Barnes ME, Gersh BJ, et al. Left atrial volume as a morphophysiologic expression of left ventricular diastolic dysfunction and relation to cardiovascular risk burden. Am J Cardiol 2002;90:1284-9.

29 Douglas PS. The left atrium: a biomarker of chronic diastolic dysfunction and cardiovascular disease risk. J Am Coll Cardiol 2003;42:1206-7.

30 Pritchett AM, Mahoney DW, Jacobsen SJ, et al. Diastolic dysfunction and left atrial volume: a population-based study. J Am Coll Cardiol 2005;45:87-92. 